\title{
DESEMBARCO DE LA ESCUELA NUEVA EN BUENOS AIRES: HETEROGÉNEAS NAVES ATRACAN EN PUERTOS HETEROGÉNEOS
}

RESUMEN

\section{ABSTRACT}

\section{- HÉCTOR RUBÉN CUCUZZA}

Universidad Nacional de Luján
El artículo tiene como objetivo de origen corroborar la referencia de Lourenço Filho sobre un viaje trasatlántico de Decroly a la Argentina en 1915, interrogante que orientó la metodología de análisis de bibliografia y fuentes primarias sobre las características de la escuela nueva. Se apoya en Marcelo Caruso cuando caracteriza al escolanovismo como una nave sin puerto definitivo, para proponer, complementariamente, la imagen de varias y heterogéneas naves arribadas a puertos a su vez diversos e internamente heterogéneos. Le cabe la metáfora de la navegación porque la construcción del movimiento internacional, en buena medida, fue resultado de los contactos surgidos de viajes realizados por sus integrantes en Europa y Estados Unidos, desde allí a Sudamérica y viceversa; durante el período de fines del siglo XIX a primeras décadas del siglo XX. A partir de las diversas y encontradas recepciones que tuvieron las ideas de Decroly en Argentina y Colombia, se analiza en particular el caso en Argentina, con apoyo en la publicación El Monitor de la Educación Común.

Palabras clave: Escuela Nueva. Decroly. Argentina

\section{LANDING OF THE NEW SCHOOL IN BUENOS AIRES:}

\section{HETEROGENEOUS SHIPS IN HETEROGENEOUS PORTS}

The original aim of this article is to corroborate the reference of Lourenço Filho about a transatlantic journey made by Decroly to Argentina in 1915, question that guided the methodology of the analysis of the bibliography and primary sources about the new school. It is based on a quote from Marcelo Caruso when he caracterizes the escolanovismo like a ship without a final port, to complimentary propose the image of multiple and heterogeneous ships arrived to ports that are at the same time, internally heterogeneous and diverse. It fits the metaphor of the navigation because the construction of the international movement was, in good measure, the result of the contacts that arose from the trips made by their members in Europe and 
in The United States, and from there, to South America and vice versa. From the diverse and controversial receptions that had the ideas of Decroly in Argentina and Colombia, we particularly discussed the case in Argentina, with the support of the publication of the Monitor of the Common Education.

Keywords: New School. Decroly. Argentina

\section{RESUMO DESEMBARQUE DA NOVA ESCOLA EM BUENOS AIRES: NAVIOS HETEROGÉNEOS EM PORTOS HETEROGÊNEOS}

O artigo tem como ponto de partida corroborar a referência de Lourenço Filho a uma viagem transatlântica de Decroly à Argentina, em 1915, questão que orientou a metodologia de análise da literatura e de fontes primárias sobre as características da Escola Nova. Baseia-se em uma citação de Marcelo Caruso, que caracteriza o escolanovismo como um navio sem porto definitivo, e propõe a imagem complementar de vários e diferentes navios atracados a portos, da mesma forma, heterogêneos e diversificados. Cabe a metáfora da navegação porque a construção desse movimento internacional, em boa medida, resultou dos contatos decorrentes de viagens feitas por seus integrantes, na Europa e nos Estados Unidos, e de lá para a América do Sul e vice-versa, entre o final do século XIX e as primeiras décadas do século XX. A partir da acolhida diferenciada que as ideias de Decroly tiveram na Argentina e na Colômbia, segundo as fontes escontradas, este artigo analisa, em particular, o caso da Argentina, com apoio na publicação El Monitor de la Educación Común.

Palavras-chave: Nova Escola. Decroly. Argentina.

\section{Cuestiones introductorias: naves parten de Europa y EEUU}

Marcelo Caruso interroga ya en el título de su trabajo acerca de la escuela nueva como ¿una nave sin puerto definitivo? (CARUSO, 2001); apoyándose en una metáfora original de Lorenzo Luzuriaga:

"La nave de la nueva educación debe seguir navegando. No hay para ella puerto definitivo, como no lo hay para nada en la historia humana. La nueva educación no es algo estático y conquistado de una vez y para siempre sino que es un movimiento, una tendencia constante hacia ideas y métodos cada vez más perfec- tos" (LUZURIAGA, citado por CARUSO, op.cit., pp.129-130)

La metáfora navegante atraviesa la estructura este artículo donde trabajaremos con los intercambios "educativos" de ida y vuelta entre centros y periferias ocurridos entre las últimas décadas de fines del siglo XIX y las primeras del XX centrándonos en Ovide Decroly.

En este período demarcado la expansión eurooccidental que arrancara en el siglo XV alcanzará su máxima expresión con la confor- 
mación de los sistemas educativos nacionales en expansión en el período de entreguerras, para finalmente erigir a la escuela en el modo hegemónico de transmisión de saberes culminando lo que hemos venido denominando proceso de escolarización de la cultura. (CUCUZZA, 2011)

La internacionalización de la escuela era acompañada por la del carbón, del hierro, de la electricidad, del capital, en fin, de las cañoneras de la colonización imperialista y de sus resistencias. $Y$, en relación con las políticas de protección infantil, se irá consolidando desde la celebración del primer Congreso Internacional de Protección a la Infancia, Paris, 1883. (DÁVILA BALSERA y NAYA GARMENDIA, 2006, p. 74).

La escuela nueva formó parte de este proceso de "globalización" a nivel euroatlántico, en el marco de una internacionalización de la problemática educativa en general, como señalan Suasnábar y Cheli:

“[...] entre 1860 y 1910 se realizaron más de 250 encuentros, conferencias y congresos en Europa y Estados Unidos que focalizaban en distintas temáticas desde la educación en general, educación moral y el niño, hasta eventos de psicología y filosofía que incluían el problema educativo, y más específicos de pedagogía (pedología, pedagogía experimental) o ciencias de la educación." (SUASNÁBAR, y CHELI, 2015, p. 93)

En 1921 en el Congreso de Calais, se creó la Liga Internacional de la Educación Nueva, donde se aprobaron los famosos treinta principios de construcción de la identidad escolano vista.

\footnotetext{
“La Liga se propuso introducir en la Escuela su ideal y sus métodos educativos, establecer una cooperación más intima entre los educadores que se adhieran a sus principios mediante Congresos, que se programaron bianuales y a través de Revistas, de las cuales destacaron tres: Pour l'ére nouvelle en Ginebra para las naciones de habla francesa, fundada por A. Ferrière; The new Era, en Londres, dirigida por Beatrice Ensor, destinada a los países de lengua inglesa, y Das Werdende Zeitelter, en Berlín, creada por
}

E. Rotten, para los de lengua alemana." (MARÍN IBÁÑEZ, 1976, p. 27)

Marcelo Caruso propone un "catálogo" abarcador de tanta heterogeneidad a partir del concepto de "principios de determinación curricular"; aclara que "no es tanto exhaustivo como aproximativo a la variedad de principios curriculares en danza"; y expresamente manifiesta que "se han dejado de lado algunas experiencias" como el plan Jena, Gentile, Spranger, Cousinet, Freinet y "fundamentalmente la poderosísima pedagogía de John Dewey [...] que merece un estudio aparte". (CARUSO, op. Cit. p. 104-105).

El catálogo señala características e integrantes sobre a) Las pedagogías renovadoras (Tolstoi, Reddie, Desmolins), b) Las pedagogías de anclaje naturalista-biologista (Montessori, Decroly), c) Las pedagogías individualizantes ( Claparède, Bovet, Ferrière, Piaget), d) Las pedagogías comunitaristas (Lietz, Wyneken, Geheeb, Powell), e) Las pedagogías del trabajo (Seidl, Kerschensteiner, f) Las pedagogías pragmáticas ( Parkhurst, Dewey, Kilpatrick, Washburne) (Ibid. p. 104-124).

Caruso comienza a considerar en el apartado "De la variabilidad a la regularidad" [...] que las posiciones antemencionadas se presentan "diversificadas de manera inusitada en un movimiento que pretende algún tipo de unidad" para destacar que "ante la expansión de alternativas organizacionales, psicopedagógicas y didácticas es necesario preguntarse qué elemento da unidad a la escuela nueva más allá de su nombre" (Ibid. p. 124-123.).

Se cuestiona luego las características de "una definición algo limitada como la del reconocimiento de la organización internacional"; y aquí vale mencionar cómo obtiene Decroly ese reconocimiento según Pozo Andrés:

"Decroly no fue el inventor del término «centros de interés» [el que] no aparece en sus escritos hasta 1921, año en el que, con el apoyo de algu- 
nos de sus colaboradores más cercanos, . Las conferencias pronunciadas en ese evento por él mismo y por Amelie Hamaïde; la publicación, junto con Gerard Boon, de la obra Vers l'école rénovée y de un artículo en la Rivista de Psicologia [...] le proporcionaron, no sólo la fama como pedagogo, que ya la tenía, sino también la gloria de apadrinar y denominar un método didáctico, el método Decroly..." (POZO ANDRÉS, 2006, p. 146. Cursivas propias)

Volviendo a Caruso, repasa las posiciones de tres marxistas que intentaron responder al interrogante acerca de la unidad en la heterogeneidad señalada visitando las posturas de Antonio Gramsci, Dermeval Saviani y Aníbal Ponce; reconoce en el primero su "agudeza" en la consideración de la escuela "tradicional" como "jesuítica", entre otros conceptos; critica al segundo por la justificación leninista de la "curvatura de la vara" de sus posiciones sobre la escuela nueva "como una recomposición de la hegemonía dominante en el Brasil de los años veinte y treinta"1; y se refiere al último como "un marxista de tempranas intuiciones reproductivistas" al considerar a la escuela nueva como "el ajuste de la pedagogía a las nuevas etapas de intensificación de la sociedad capitalista" (Ibid., p. 125-127). ${ }^{2}$

Y formulará su hipótesis del carácter de la escuela nueva como "reforma productiva (y protestante) de las pedagogías triunfantes":

"Como cada pedagogía triunfante es diferente según la historia de cada contexto, las respuestas que buscaban la productividad de lo educativo y la consideración de la naturaleza infantil -lo que hemos denominado como una forma de protestantismo cultural- adquirió formas también diferenciadas en los diversos países" (Ibid. p. 129).

Aquí nos interesa agregar que, tanto así como "escuela nueva" no es un bloque ho-

1 Véase su versión del uso de Lenin en GONÇÁLVES VIDAL, 2011. pp. 43-45.

2 Agregaríamos aquí, que Aníbal Ponce se apoyaba en el marxismo de la década de los años ' 30 y no tuvo conocimiento de Gramsci por su muerte temprana. mogéneo tampoco lo es "escuela tradicional", ambos términos de algunos cuadros de doble entrada que suelen reducir complejidad.

Apoyándose en los análisis de Anne Querrien sobre el método simultáneo y el lancasteriano, Caruso afirmará que "la escuela «tradicional» de la modernidad (aunque suene a paradoja) no es positivista, como se suele afirmar, sino que el positivismo parece entablarse como reformador - en muchos casos autoritario - de las decisiones didácticas ya tomadas bajo la influencia de las formas disciplinarias derivadas de las escuelas religiosas" (Ibid. p. 128).

En la misma línea del análisis en tiempos más largos, Marc Depaepe y Frank Simon también se aproximan a esta identificación del polisémico "escuela tradicional" con la permanencia del sistema jesuítico: “...los jesuitas ya estaban presentes en la creación de la gramática contemporánea de la escolarización y de la pedagogización" (DEPAEPE y SIMON, 2008. p. 112.)

Para cerrar este apartado, cabe señalar que no encontramos factible aplicar el "catálogo" de Caruso a la escuela nueva en sus expresiones en nuestro país; debido fundamentalmente a que las experiencias locales se manifestaron como heterogéneas adaptaciones con diversos grados de eclecticismo.

En palabras de Sara Alí Jafella:

"Cabría afirmar que los principios de este movimiento educativo se manifestaron de manera asistemática, fragmentaria, sin atender a un programa racionalmente organizado, pero estuvo presente - y quizá omnipresente- como una forma de underground pedagógico" (ALI JAFELLA, 2004. p. 358, subrayado propio).

\section{Los puertos sudamericanos de Colombia y Argentina}

Hemos encontrado versiones contrarias en alguna bibliografía acerca de los viajes de Decroly a Latinoamérica. Véanse las siguientes: 
“Tuvo eco en los Estados Unidos, donde Decroly fue llamado en 1922; en Bolivia, Argentina y Uruguay donde el gran maestro estuvo en 1915; en España, donde realizó un curso especial al año siguiente" (FILHO L., 1964, p. 201)

“...sus ideas alcanzaron otros países, habiendo visitado algunos de ellos, tales como Uruguay, Bolivia, Argentina (1915), los Estados Unidos (1922), Colombia (1925) y España" (HERRERA CORTÉS, 1999, p. 54)

"[varios viajes entre los cuales] El viaje que llevó a cabo en 1925 en la América Latina donde entró en contacto con los medios pedagógicos renovadores de Uruguay, Colombia, Bolivia, Argentina y Brasil" (SEGERS, 1985, p. 19)

No abundan los estudios comparados sobre la escuela nueva en Colombia y Argentina aunque sí se disponen de trabajos sobre Argentina y Brasil. Mencionamos por su carácter precursor la compilación que dirigiera Silvina Gvirtz: Escuela Nueva en Argentina y Brasil (1997).

Un estudio más reciente afirma que los rasgos de similitud entre Brasil y la Argentina con relación a ese enfoque pedagógico provienen de diversas corrientes:

“...en el caso de Brasil fue el pensamiento norteamericano, especialmente Dewey, el más representativo. En la Argentina, en cambio, la influencia fue más fragmentaria y registra principios educacionales provenientes de Ovidio Decroly, María Montessori y de la línea anglosajona, tanto la oriunda de Inglaterra [...] como la sustentada por el pragmatismo y la filosofia educacional de Dewey..." (ALI JAFELLA, S. 2004, p. 355)

En cuanto a Colombia es clara la acogida generalizada hacia Decroly en 1925. Los textos recogen la invitación del fundador del Gimnasio Moderno de Bogotá en 1914 así como sus contactos previos en Europa. (OBREGÓN et al., 1997, p. 321/ HERRERA CORTÉS, 1999, p. 57/ RÍOS BELTRAN, 2012, p. 95).

“Por invitación de Nieto Caballero, el pedagogo belga Ovide Decroly visitó Colombia durante dos meses en 1925. Dictó una serie de conferencias en el Gimnasio Moderno, cuyo objetivo era orientar la tarea del examen mental y escolar por medio de los tests individuales y colectivos, así como la selección, la orientación profesional y el tratamiento de los niños excepcionales." (OBREGÓN, et al, 1997. p. 321)

Dichos autores ofrecen algunas respuestas a la amplia recepción de las ideas de Decroly en Colombia entre 1925 y 1935, a saber: a) la crítica a los métodos de la escuela tradicional, b) la cercanía de su concepción del conocimiento y los métodos de enseñanza con la práctica pedagógica vigente en el país, c) el énfasis decrolyano en la educación como adaptación social [y con ello] a las estrategias de formación en el país a la "cultura católica" de la nación, d) la centralidad en todo el sistema decrolyano del examen y la clasificación escolar, así como de la selección y educación especial de anormales, e) y por último, el biologicismo y el darwinismo social que fundamenta sus ideas pedagógicas. (Op. cit., p. 330-331)

Vale extenderse en el último punto cuando los autores señalan que ese biologicismo se expresaba en:

“...la continuidad entre selección natural y selección social y en la importancia de la armonía social fundamentada en el postulado de ubicar socialmente a cada individuo de acuerdo con el determinismo de la ley de la evolución; nociones que también hacian parte de los discursos sobre la degeneración de la raza, y la implantación de una Escuela Defensiva en el país" (Ibid., p. 331$)^{3}$

En cambio, una heterogénea recepción tuvieron las ideas de Decroly en Argentina ex-

3 "La unión entre la eugenesia y la higiene social podía ser considerada una consecuencia de la influencia creciente del darwinismo social, por la que se limaban los afilados bordes de la eugenesia. La influencia del darwinismo social había ocasionado por lo tanto un desplazamiento del objetivo original, que aspiraba a la higiene corporal y mental del individuo, hacia este nuevo objetivo social. La adaptación individual con Decroly se ponía al servicio de la adaptación de la especie. (DEPAEPE, SIMON y VAN GORP, 2010. p. 24) 
presada en diferentes posturas y adaptaciones eclécticas. Señala Carli:

"En Buenos Aires, las ideas de la escuela nueva circularon "en forma contemporánea al crecimiento del socialismo, del anarquismo, del liberalismo democrático, del comunismo, etc.; no obstante, también se combinaron con discursos orientados hacia la moralización de las costumbres sociales en el pensamiento de ciertas figuras que una década después se posicionaron junto con el nacionalismo. Nos referimos a figuras como la pedagoga Clotilde Guillén de Rezzano y al pedagogo Hugo Calzetti" (CARLI, 2004, p. 365)

Frente a tanta diversidad política e ideológica Adriana Puiggrós propuso en su momento una clasificación de los normalistas solidaria con sus propias concepciones acerca del papel del sistema educativo argentino en sus orígenes.

La propuesta ampliamente difundida en ponencias, tesis de maestrado y doctorado, publicaciones diversas en el campo de las ciencias de la educación en especial; partía de una clasificación de los egresados de la escuela normal como normalizadores católicos, normalizadores laicos, democrático radicalizados, socialistas y anarquistas (PUIGGRÓS, 1990, p. 41-42. Cursivas propias)

Siendo que los escolanovistas argentinos eran normalistas resulta cuasi natural la aplicación de la clasificación a la escuela nueva y sus integrantes, aunque todo cuadro clasificatorio se desdibuja en cuanto los estudios particulares agregan complejidad en los casos que se encuentran en los bordes.

Véase el trabajo de Inés Dussel (2014, p. 3) sobre Mercante y las referencias que realiza sobre estudios recientes de Lucía Lionetti [la que] "discute implícitamente a Puiggrós en su visión «un tanto simplista y unilineal» de la relación entre los normalizadores y las políticas estatales" [sic].

Por otra parte, la clasificación adopta un doble criterio entre las dos primeras entradas cuasi funcionales y las dos últimas expresamente referidas a encuadres políticos y aquí agregamos que, en este último caso faltarian los comunistas, por ejemplo, la paradigmática Florencia Fossatti.

En el tomo tercero de la trilogía inicial de su Historia de la Educación Argentina de Galerna, en la dirección de rescatar la complejidad del movimiento escolanovista en Argentina, Adriana Puiggrós caracterizó distintas posiciones cuyo eje común sería la oposición al "modelo normalizador". Estas serían: 1) las posiciones «orgánicas», 2) las posiciones «transgresoras» y 3) la corriente «radicalizada» (PUIGGRÓS, 1992, p. 62-64).

Esta clasificación aplicada a "las corrientes activistas y pragmatistas", y sobre todo sus relaciones con la anterior clasificación de los normalistas, merecería un análisis más extendido que el permitido por esta síntesis. No obstante destacamos algunas observaciones de la propia autora que relativizan la clasificación, a saber:

“El agrupamiento de las corrientes pedagógicas de la época en «positivistas» y «antipositivistas», así como la definición global- a favor o en contra- de la escuela activa argentina oscurecen la comprensión de los acontecimientos."[...]

“elementos provenientes del positivismo y en particular de tono krausopositivista, pervivieron en los discursos de casi todos los educadores progresistas de la época, incluídos los "activistas» declarados, de orientación espiritualista".

"el conjunto de los educadores «pragmatistas» y «"activistas»" provenían del espacio del normalismo y reflejaron las transformaciones que sufrieron los discursos que lo poblaban." ( PUIGGRÓS, 1992. p. 59)

Agrega que "la superposición, distinción o antagonismo de los planteos «activistas» con los «normalizadores» permitirian “agrupar a los educadores de acuerdo a coincidencias y diferencias", para concluir que "pierde perti- 
nencia la denominación de «escuela activa» 0 «nueva» y pragmática como una corriente y se abre como campo problemático el conjunto de las propuestas alternativas al modelo normalizador". Y relativiza la clasificación cuando añade que "caracterizaremos distintas posiciones dentro de aquel campo que no son excluyentes entre sí y en las cuales en ocasiones son intercambiables los personajes que tomamos como ejemplo" (Ibid. p. 60) (PUIGGRÓS, 1992, p. 60. Subrayado propio)

La amplia producción de Adriana Puiggrós obliga al seguimiento de sus propuestas teóricas más complejas, y en este caso, en una publicación más reciente, abre sus propios encuadres para recoger la heterogeneidad apoyándose en trabajos propios y de otros autores como Sandra Carli para señalar las:

“...diferencias que existieron entre los escolanovistas adeptos a las ideas de Pestalozzi y aquellos influidos por anarquistas y socialistas. Entre los primeros está José Rezzano, con la revista La Obra bajo su dirección. Entre los segundos, militantes comunistas como la mendocina Florencia Fossatti y el uruguayo Jesualdo, anarquistas como Julio R. Barcos y socialistas como Olga Cossettini. Se agrega, como una tercera variante, el escolanovismo nacionalista popular de Saúl Taborda (1885-1944)." (PUIGGRÓS, 2003, p. 39).

Y cerraremos este apartado con las interpretaciones que realiza Carli sobre sus señalamientos sobre la construcción de un conjunto de equivalencias respecto del discurso fundador de la instrucción pública, que hizo notoria la confrontación entre normalismo positivista y escolanovismo:

"Estas equivalencias eran, entre otras, paidocentrismo-puerocentrismo; enseñanza-aprendizaje; disciplina escolar-autogobierno infantil. El trabajo de diferenciación operado por el discurso de la escuela nueva no redundó en la construcción de una nueva hegemonía político-educativa que modificara los cimientos del sistema escolar" (CARLI, 2003, p. 189).

\section{Una temprana encalladura: la} investigación sobre los viáticos

Nos referimos a la investigación llevada a cabo en el Consejo Nacional de Educación apenas asumido como presidente José María Ramos Mejía en 1908. En el informe que a su pedido le envía el contador Juan R. Silveira el 31 de marzo de 1908, señala que le han llamado la atención "las crecidas sumas que han sido acordadas a algunas maestras y maestros para emprender viajes fuera del país", adjunta un listado con nombres e imputaciones presupuestarias para agregar en la suma final:

“Pasajes abonados a la agencia de los señores Antonio M. Delfino y hermano, cinco pasajes de ida y vuelta a Hamburgo, para don José Rezzano, doña Clotilde de Guillen y la madre de ésta, y doña C.C. Alvear y hermana.

\section{$\$ 346587$}

Total \$24.530 47"

(El Monitor de Educación Común, 1908, p. 484485.)

El estudio de Silveira fue pasado al vocal del Consejo Delfín Jijena quién eleva un informe al Presidente el 29 de mayo de 1908 respondiendo a cada una de las observaciones realizadas y refiriéndose a los docentes imputados señala claramente el viaje hacia los "centros de civilización escolar" (sic) de donde provenían las naves de la escuela nueva:

"El Consejo Nacional como una medida de estimular al personal docente, y a la vez, para obtener datos concretos que se relacionen con el mayor progreso técnico en la educación, ha enviado en estos últimos cuatro años, a cinco profesores a Estados Unidos y a Europa, para que hagan estudios especiales en aquellos centros de civilización escolar, por temporadas cortas, de acuerdo con los estudios que se les confiara" (Op.cit. p. 496. Subrayado propio)

El informante añade que "el Consejo les ha asignado pasajes y sobresueldo necesarios para sus viajes de estudio, ascendiendo 
el gasto en cuatro años a pesos moneda nacional $24.530,47$ sobre lo cual llama la atención el señor Contador interventor"; y presenta un listado de los beneficiados con sus respectivos cargos:

"Señor José Berrutti, dirige la escuela modelo "Presidente Roca"

Señorita Ernestina López, está al frente del primer Liceo Nacional de Señoritas en la Capital

Señorita Cármen Champy Alvear, es directora de la escuela normal nacional de reciente creación en Flores

Señorita Clotilde Guillén, es inspectora de trabajos manuales y economía doméstica de las escuelas de la Capital

Señor José Rezzano, dirige una escuela superior en el 5o. Consejo escolar" (Op.cit. p. 497, cursivas en el original)"

El trámite alcanza hasta junio de 1908 cuando José María Ramos Mejía firma el "publíquese y archivese", es decir 6 meses de burocracia en el gobierno escolar que anunciaban el giro disciplinador que adquiriría su gestión ya en sus comienzos.

Nos detenemos en los dos últimos mencionados en el informe de la investigación para realizar algún desarrollo sobre los Rezzano, ampliamente mencionados en la historiografia argentina sobre la escuela nueva.

Encontramos en una bibliografia algunas referencias sobre Clotilde Guillén a la que encontrarán firmando después de 1930 con su apellido de casada, Clotilde Guillén de Rezzano, su compañero de viaje.

"En ese mismo año se funda la Asociación de Psicología, que en sus aspectos reglamentarios establecía que el cuerpo de miembros titulares no podía exceder a cuarenta, mientras que el carácter de miembro suplente era ilimitado. Entre los primeros se encuentran "los fundadores de los primeros laboratorios, los directores de las primeras publicaciones afines, reconocidos personajes de la cultura, y una única represen- tante del género femenino, Clotilde Guillén, pedagoga de 28 años de edad." (OSTROVSKY, 2008, p. 57-58)

La autora mencionada reseña algunos datos de Clotilde Guillén como normalista que había continuado su formación en la Facultad de Filosofía y Letras (UBA), la que se había convertido en una vía de acceso a la universidad para las mujeres por ser la única que aceptaba egresados de escuelas normales sin exigir título de bachiller.

Data en el año 1906 un viaje a Europa para observar la estructura y organización de las escuelas primarias comisionada por el Consejo Nacional de Educación que la investiga en el episodio que comienza este apartado.

Continúa Ostrosvky registrando que "a su regreso se desempeña como Inspectora de Escuelas Primarias hasta 1909, primer año de funcionamiento de la Sociedad, y es nombrada directora y profesora de Pedagogía y Psicología en la Escuela Normal № 5 de Barracas y que intervino en los cursos de Pedagogía de la Facultad de Filosofía y Letras, y en la estructuración del primer ciclo primario, centrándose su labor en cuanto a la enseñanza de la psicología principalmente en el nivel medio o secundario" (OSTROVSKY, op. cit., p. 60-61).

En cuanto a sus publicaciones se advierte una nula participación en la Sociedad que se contrarresta con la mayor participación en El Monitor de la Educación Común donde se registran trabajos como "Enseñanza de la costura en las escuelas primarias elementales de Francia [...], Bibliografía de economía doméstica [...], Enseñanza de la cocina en la escuela primaria [...] o Algunas observaciones sobre el funcionamiento de las clases de cocina [...]". (Ibid.).

Es preciso "historizar" este tipo de contenidos en el contexto del llamado feminismo de primera ola que enarbolaba la lucha por el sufragio femenino aunque no cuestionaba las 
posturas sobre la "naturaleza femenina", registrándose su desempeño como presidenta de la Sección Educación del III Congreso Femenino Internacional de $1928 .{ }^{4}$

En cuanto a José Rezzano, si bien su accionar escolanovista es conocido como la Reforma Rezzano, atribuyéndole el papel como "su principal ideólogo" desde el inicio de su gestión como Inspector Técnico General en la Capital en 1918, advierte Ignacio Flechter que la iniciativa reconoce otros participantes:

"Ubicamos el epicentro de la Reforma a partir de 1920 en el Consejo Escolar № 1, con la participación fundamental de Juan C. Vignati en su implementación, al asumir en ese año como Inspector del distrito. De aquí, los dos nombres con los que se la identifica: «Sistema de labor y programas del Consejo Escolar 10®» o «Reforma Rezzano»". (FLECHTEL, 2013. Mimeo)

Entre los múltiples desempeños de Rezzano mencionaremos los de maestro, director y supervisor en escuelas dependientes del Consejo Nacional de Educación. Dirigió la revista La Obra, que diera amplia difusión al escolanovismo, fue representante de la Liga Internacional para la Nueva Educación en Argentina y creó su órgano la revista Nueva Era.

Parafraseando a Aníbal Ponce cuando decía que "en la base de la nueva técnica del trabajo escolar está Ford y no Comenio"5, en la base de la Reforma Rezzano está Taylor y no Comenio. Como destaca Gvirtz:

“[el sistema de trabajo industrial] y su comparación con los procedimientos aún en uso en el

4 Ostrovsky se apoya en estos últimos antecedentes para diferir con Adriana Puiggrós y Sandra Carli que "la ubican en la siguiente década, en una postura espiritualista y moralizante, que curiosamente coincide la inclusión de su apellido de casada en los escritos de su autoría, y testimonia un viraje hacia posiciones solidarias al conservadurismo político del Consejo Nacional de Educación hacia los años 30" (Ibid. p. 61)

5 PONCE, A., 1974, p. 427. Aunque quizá convendría señalar que el Comenio que pedía configurar la escuela como una "tipografía viviente" si estaba en la base de la escuela en los origenes manufactureros y la imprenta como paradigma de la producción. trabajo escolar, demuestran claramente cómo este último se encuentra todavía en un período en que impera el desperdicio de material y de energías por parte de docentes y alumnos y en el cual no se halla establecido un nexo lógico entre sus elementos: los que dirigen, los que vigilan, los maestros y profesores, los alumnos y los horarios, etc" (REZZANO, J. La organización del trabajo escolar de acuerdo con nuevos principios, citado por GVIRTZ, 1997. p. 3)

La propuesta de Rezzano del cuaderno único apuntaba a lograr un instrumento de control más eficiente, esto es, "Este último debió perder su característica de elemento decorativo destinado a impresionar por su presentación cuidada, detalles caligráficos, sus mapas y sus ilustraciones, fruto de horas extraordinarias de trabajo escolar o casero, sin conexión con la tarea educativa e instrucción diaria para pasar a ser un elemento de ejercitación y de trabajo en el que, hora por hora, aparece la colaboración del alumno con el maestro" (REZZANO, J. La organización del trabajo escolar de acuerdo con nuevos principios, citado por FLECHTEL, I. Op. Cit.)

En enero de 1933 se realizaron "Los cursos de Escuela Activa para maestros" en el salón de actos de la Facultad de Filosofía y Letras de Buenos Aires; bajo la organización de la Liga de la Nueva Educación presidida por el decano de la Facultad de Humanidades y Ciencias de la Educación de La Plata, Dr. José Rezzano; y el Instituto de Didáctica de la Facultad de Filosofía y Letras de Buenos Aires que dirigía el Dr. Juan E. Cassani.

José Rezzano pronunció el discurso inaugural refiriéndose a la escuela nueva como "un fenómeno de carácter universal [que] en el último Congreso celebrado en Niza en agosto último [mostró a] observadores imparciales cómo por sobre la impresionante diversidad aparente de delegados llegados de todo el mundo, de religiones y sectas diversas, de idioma distinto, de ideologías filosóficas, po- 
líticas, sociales y económicas contrapuestas, flotaba un espíritu unificador del cual derivaba la convicción de que fueran cuales fueran las posiciones tomadas en otros campos, la escuela nueva tenía que ser para todos el instrumento necesario para ayudar a la humanidad a salvar los escollos y peligros del momento actual" (MEC. 1933, p. 100).

Tanto la atmósfera belicista general del occidente de entre guerras como en especial de nuestro país en la década del '30, más conocida como de la "restauración oligárquica conservadora"; podría explicar este ademán salvacionista; pero, el conservadurismo nacionalista y sus políticas de persecución ideológica no autorizaban semejante amplitud.

Esto explica las consideraciones "nacionalistas" que ofrece Rezzano respecto de los fines de los cursos:

“El objetivo concreto a que apuntan estos cursos es el de facilitar en forma ordenada y sistemática la iniciación de parte de nuestros maestros de esa tarea nacional, vale decir, propia de nuestro pueblo, que llegue en definitiva a darnos la elaboración y la aplicación de un sistema educativo encuadrado en los principios generales de la nueva educación, pero con las características y relieves propios de nuestra personalidad nacional" (Ibid, p. 102. Subrayado propio)

Anuncia contenidos de los cursos sobre "algunos sistemas nuevos: el de Decroly, el Plan Dalton y el Plan Jena" y vuelve a reforzar su "nacionalización" advirtiendo que ellos "serán apreciados objetivamente pero vistos y estudiados con ojos y criterios de maestros argentinos" (Ibid. p. 103. Subrayado propio)

Siguió una referencia a Vasconcelos en el espinoso tema de la "antinomia de la libertad y de la autoridad", la cuestión de la disciplina, que venía enfrentando a "normalizadores" y "radicalizados" y comenzaron los cursos con los siguientes docentes:
- Dr. Juan E. Cassani, “Los tests en la escuela primaria"

- Prof. Oscar Tolosa, “El plan Dalton y nuestra escuela"

- Clotilde Guillén de Rezzano,"Los centros de interés y la técnica de la escuela activa"

- José Mas, “Renovación de nuestro trabajo escolar"

- Juan Mantovani, "El Plan Jena y la reforma escolar alemana"

- Salvador Aloise, "Algunas anomalías psíquicas del escolar"

- José Rezano, “La didáctica de la escuela nueva"

Tanto los contenidos como los docentes demuestran la existencia de un grupo crítico escolanovista y espiritualista que ya ocupa cargos de relevancia marcando un largo recorrido con aquel episodio de la investigación de 1908.

Hubo un cierre con salutaciones diversas y "finalmente la señora de Rezzano, declaró constituída con los presentes la Liga de la Nueva Educación, adherida a la Liga Internacional, la que editará un boletín informativo mensual" (Ibid, p. 106. Cursivas propias)

\section{Vientos de proa rotan a vientos de popa: visiones encontradas sobre Decroly}

Siguiendo a Sandra Carli, en la Argentina fue muy clara la "divisoria de aguas" en los años 30 entre los simpatizantes del régimen militar-nacionalista encabezado por el general José Félix Uriburu y sus opositores. (CARLI, 2004, p. 365)

Nos detendremos en este apartado en la consideración de dos posturas acerca de Decroly, en especial, porque demuestran claramente las posiciones antagónicas que pudieron visualizarse a partir del golpe de estado 
de 1930 que derrocó a Hipólito Irigoyen, primer presidente constitucional electo por el voto secreto y obligatorio.

En el primer caso nos referiremos al Dr. Ángel Acuña, quién venía con varios desempeños como vocal en el Consejo Nacional de Educación y como vicepresidente del Dr. Emilio Giménez Zapiola en ese cuerpo, reemplazándolo interinamente en la presidencia a partir de su fallecimiento en setiembre de 1930.

En el discurso de Acuña pronunciado en el acto del sepelio expresa claramente sus posturas en favor del gobierno de facto y sus consideraciones sobre el irigoyenismo afirmando que, en la sociedad argentina se evidenciaba "la salida de los cauces legales, la ausencia de gobierno en las funciones directivas, la descomposición en los organismos del Estado, la atrofia del sentido moral" y añade que "El fermento se extendía a todos los resortes de la administración y amenazaba atacar en sus entrañas la vida misma de la nacionalidad"; mientras que el gobierno surgido del golpe de estado de 1930, se proponía “devolver al país la normalidad" y "restablecer dentro de la constitución y de la ley, la función de gobierno" (ACUÑA, 1931, p. 13)

Luego de su interinato, asumió la presidencia del Consejo Nacional de Educación el Dr. Juan B. Terán quien, siguiendo a Adriana Puiggrós, era "un espiritualista, antilaicista y conservador" que "daba el tono del clima cultural de la época" (PUIGGRÓS, 2003, p. 117).

El Monitor publica dos artículos de Acuña sobre Decroly ambos en el año 1934, el primero de tipo descriptivo para el que remitimos a la fuente primaria por razones de sintesis y el segundo que subtitula "Estudio crítico" en el que nos detendremos por cuanto representa posiciones contrarias a la recepción de Decroly. El trabajo le lleva más de ocho páginas y transcribiremos los puntos principales de sus observaciones críticas, a saber:
1) No existe algo que pueda definirse como "mentalidad infantil"

“Decroly, como los otros dogmáticos de la escuela, parte del punto de vista de la igualdad del espíritu humano, de la absoluta identidad de las inteligencias, y en consecuencia, de la afirmación del principio de que existe una «mentalidad infantil», siempre igual, y un exclusivo mecanismo de funcionamiento intelectual. Tal es en nuestro concepto su error" (ACUÑA, (2) 1934, p. 3)

2) No existe la aptitud de la globalización en el niño

“La doctrina, encuentra, pues, un obstáculo irrecusable en su parte sustancial: la vulnerabilidad de la base compromete la solidez de su construcción, Los estudios de psicología infantil niegan la existencia de semejante aptitud de «globalización» en el niño, afirmando al contrario, que la mentalidad infantil se caracteriza por la percepción del detalle y la dificultad de la visión de conjunto" (Ibid, p. 4)

3) La adquisición del lenguaje oral difiere del aprendizaje de la lectura y escritura “Es cierto que la adquisición del lenguaje se hace en el niño por palabras, no por letras y sílabas, pero también es innegable que es la forma habitual del aprendizaje de la lengua; la descomposición se verifica en la enseñanza de la lectura o la escritura, cuando se impone el dominio del abecedario y el conocimiento preciso de las letras" (Ibid, p. 5)

4) La creación de los centros de interés de Decroly perturba la adquisición científica “Pero la base fundamental de su reforma radica en la creación del sistema de «los centros de interés». Modifica sustancialmente con ellos el régimen de la organización de la enseñanza y toca en su esencia las bases mismas del saber. Es cierto que su aplicación está limitada a pocos años de estudio, pero lo suficiente para crear hábitos mentales que perturban la aptitud de la adquisición científica, que debe formarse desde niño" (Ibid, p. 7) 
Veamos ahora un artículo en favor de Decroly de Bernardina Dabat de López Elitchery, escolanovista enrolada políticamente en el socialismo, titulado significativamente como "Apuntes sobre una adaptación del método «Decroly» a nuestras prácticas escolares", referido a "una experiencia realizada en las escuelas № 71 "Francisco de Godoy" y № 77 "1으 de Julio" de Rosario de Santa Fe" (DABAT de LÓPEZ ELYCHERI, 1932, p. 9)

Bajo el título general "Globalización de la enseñanza" reseña experiencias realizadas con tests en la escuela № 54 "Belgrano", a base de cuestionarios problemas en la № 78 "Francisco de Seguís", la realización de trabajos de observación, de investigación y de expresión por grupos a lo Cousinet en la № 56 “Almafuerte"; y agrega "el ensayo de adaptación del Plan Dalton de la № 90 "Capital Federal”. (Ibid., p. 9. Subrayado propio).

Citando a Dewey se refiere a formar la personalidad del niño cultivando en él "las nociones de libertad y responsabilidad"; señala la "desconfianza traduce en mucho y en muchos, un estado de miedo instintivo" como si estuviera interpelando a hipotéticos pedagogos o docentes; para destacar que dicho miedo "nos atormente un poco menos siquiera (sic) "a los que vimos a don Martín Herrera, cuando estableció, bajo las tribunas del Hipódromo de esta ciudad, la pequeña escuela "al aire libre»". A los que vimos este ensayo en el momento de su iniciación y a los que leímos con interés lo que dijo e hizo en su hora el señor Vergara" Seguimos sus palabras cuando se dispone a "encaminar nuestro «ensayo» de adaptación del método Decroly" apoyándose en las prácticas en las escuelas mencionadas al comenzar y "en lo leído y en la pequeña experiencia recogida en diversas aplicaciones parciales, a veces hasta inconscientes, de las ideas decrolyanas por nosotros mismos" (Ibid., p. 10).:
- "Todos los que hacen correlación de estudios, los que aplican el método de «ideas asociadas» están dentro de las ideas de DECROLY.

- Todos los que inician la enseñanza de la lectura-escritura con el método ideo-visual-motriz lo están igualmente en este momento y pronto tendrán que decirnos por qué razón lo abandonan; por qué no prosiguen con la «globalización» de la enseñanza en los grados subsiguientes.

- Todos los que no rompen con la disciplina formal a que estamos acostumbrados, estorban a la verdadera experiencia; no están dentro del espíritu del pedagogo belga y no pueden así recoger las lecciones que promete el maestro ni estimularlas en su verdadero valor, en su mentada eficacia" (Ibid., p.10. Cursivas de la autora)

Bajo los subtítulos "Globalización de la enseñanza”, "Cuáles son los fundamentos psicológicos del trabajo de globalización", "Práctica de la globalización entre nosotros" y "Conclusiones"; el artículo de Bernardina Dabat se extiende por 16 páginas del Monitor por lo que seguiremos el mismo procedimiento adoptado para el artículo de Acuña, en función de la síntesis, a saber:

1) Pre-existencias de prácticas y papel del maestro

“...con el uso de cuestionarios (que nos fue traído hace unos años, primer paso hacia la individualización en las formas del trabajo, propiamente dicho); con la autocorrección; con el gobierno propio...Todas estas prácticas se nos han venido colando diré, un poco subrepticiamente, hasta hacernos ver [...] que el papel del maestro es otro frente a los "grupos» en la obra de la educación moder$n a$, en todos sus grados en el concepto de la Escuela nueva" (Ibid., p. 11. Cursivas de la autora)

2) Postergación del análisis frente a la percepción global 
[siguiendo a Claparede vemos que] "...el niño no tiene ningún interés en analizar, cuando las partes del todo que observa le son desconocidas o no suscitan su interés de una manera particular. Advirtamos que esta necesidad de no precipitar el análisis es algo que interesa mucho dentro de lo que es el espíritu del método Decroly" (Ibid., p. 15. Cursivas de la autora)

3) Oportunidad de ejercitar el análisis

"El trabajo aconsejado por Decroly a base de "globalización" sigue interesando en todos los grados y asumiendo gradual y paulatinamente el carácter de analítico sintético. Porque correlativamente: no ejercitar la capacidad de análisis suficientemente y a su debido tiempo, será cometer la herejía de pretender dejar a los hombres en la condición de niños." (Ibid., p. 15. Cursivas de la autora)

Pasaremos a realizar un breve recorrido por dos "comunistas" que actuaron en períodos ligeramente diferentes: Florencia Fossatti y Anunciada Mastelli más adelante.

Florencia Fossatti lideró la huelga del magisterio mendocino de 1919 que culminó con la incorporación del gremio docente a la F.O.R.A., la organización sindical del anarquismo en nuestro país. (CRESPI, 1997, p. 155).

A partir de 1930, la Dirección General de Escuelas, autorizó la apertura de la Escuela Experimental Nueva Era, a cargo del Centro de Estudios Pedagógicos “y a la maestra Florencia Fossatti como Inspectora de Escuelas que se propuso organizar una exposición de material didáctico y de trabajos escolares, para favorecer la experiencia de la Escuela Nueva" (SÁNCHEZ LUQUEZ, 2003, p. 68).

En esa etapa, Florencia Fossatti dejó su cargo de inspectora para tomar la dirección de la Escuela Complementaria Presidente Quintana donde organizó tribunales infantiles y la aplicación del Plan Dalton, teniendo presente las advertencias que planteaba el mismo Decroly:
"Los métodos individuales y las formas de trabajo que se ensayan en las escuelas el mismo plan Dalton, si bien constituye un sistema de transición, se recomienda por esto mismo, ya que por lo menos cuando se trata de experiencias de gran alcance, se hacen necesarias estas transiciones que son etapas de una evolución más segura" (Ibid., p. 69) ${ }^{6}$

El Centro de Estudios Pedagógicos Nueva Era estaba integrado entre otros, por María Elena Champeau, Florencia Fossatti. Néstor Lemos; quiénes se formulan un ambicioso plan de difusión que desarrollan entre 1928 y 1930; publicando la revista Ensayos. (FONTANA, 1993, p. 286-287)

En 1936 tuvo lugar un debate legislativo en el que actúa como fiscal acusador el diputado conservador Edmundo Correa que culminó en la exoneración de Florencia Fossatti. El mismo realiza declaraciones al periódico Los Andes donde afirma haberse apoyado en un documento secreto del Ministerio de Guerra donde se decía que la tal activa comunista Florencia Fossatti,

“...por intermedio de los tres actuales inspectores de escuelas de la Provincia, que son sus discípulos: señorita Champeau y señores Codorniú y París, ha introducido la Enseñanza Activa y los Tribunales de Disciplina destinados a disminuir la autoridad del maestro. Un importante paso a la instrucción soviética es la instrucción sexual que se imparte a su iniciativa en los grados superiores" (FONTANA, 1993, p. 290)

Continuemos con Anunciada Mastelli quien se recibe en el año 1926 como profesora de historia y pasa a ejercer como maestra titular en 1927 en la Escuela № 17 del Distrito Escolar 20. En los suburbios de la ciudad de Buenos Aires, en el barrio de Mataderos, Anunciada realizó sus eclécticas experiencias

6 La autora toma la cita de la Revista Orientación, Año II, № 2, enero 1933, p. 2. Entrevista realizada por Luis Codorniú Almazán a Ovide Decroly en Ucle el 3 de enero de 1933. Es probable la entrevista en Ucle donde fallece Decroly en setiembre de 1932. No así su fecha por obvias razones. 
docentes bajo la inspiración de las ideas de la escuela nueva.

En la "sección pedagógica" de la revista Vida Femenina publicó dos artículos dedicados a "La Escuela Laboratorio Plan Dalton". Señala en el primero, citando a la creadora del método, Ellen Parkhurst:

\begin{abstract}
“Hoy pensamos demasiado en el programa de estudios y demasiado poco en los muchachos y muchachas. El programa es solamente una técnica, unos medios para un fin. El instrumento que debe ser manejado es el muchacho o la muchacha. [...] Todas las dificultades que agobian al maestro derivan de las dificultades no resueltas de los alumnos. Cuando las últimas desaparezcan, las primeras se desvanecerán también, pero no antes de que la organización escolar y la maquinación que le acompaña hayan sido rehechas para el alumno, quien se ha vuelto ineficaz e irritable por verse obligado a usar un mecanismo que no es el suyo propio" (MASTELLI, 1937, p. 18).
\end{abstract}

Seguidamente, da cuenta de los principios organizativos del método bajo los subtítulos "En qué consiste el Plan Dalton", "Organización de la labor", "Edificio escolar", "Material de trabajo", "Horario", "Distribución de las horas de trabajo", “Contralor del trabajo", “Programa”.

En un segundo artículo [...] se dedica a enfatizar las aplicaciones creadoras del método realizadas por docentes argentinas. Menciona, entre otras, las experiencias tempranas de Florencia Fossatti en la provincia de Mendoza y destaca también los trabajos de Ángela G. De Agüero sobre un ensayo de Plan Dalton hecho en Rosario en los años 1930, 1931 y 1932, “que tiene para nosotros el interés especial de ser una adaptación a la escuela argentina". (Ibid)

Ese "interés especial" la lleva a recoger otros antecedentes: “En la Escuela Normal № 5, donde actuó la señora de Rezzano, los grados superiores pusieron en práctica una forma «daltonizada» de trabajo y la profesora Crespo, de la Escuela Normal № 1, ha publicado ya varios cuadernos de tareas para los alumnos que ha titulado "Daltonizando la escuela" (MASTELLI, 1937, p. 37. Cursivas propias).

Esta frase de la publicación refuerza las acciones de adaptación paralelas a la "daltonización":

"Como todo método nuevo, corre el riesgo de momificarse [...] el ideal sería ensayar constantemente y tener la zozobra de que aún cabe hacerlo mucho mejor [...] y nada hace desmerecer a este ensayo, que hoy es mejor que las viejas normas, la idea de que sea superado en el porvenir. Esta inquietud es la que hace marchar al mundo" (MASTELLI, 1937, p. 31).

Anunciada Mastelli no alcanzó en nuestra producción historiográfica el espacio de otros escolanovistas, quizá porque sus prácticas gremiales y políticas de acción directa desplazaron a sus prácticas docentes de sus intereses de producción teórica.

La encontraremos exponiendo en la sesión inaugural del 4 de junio de 1943 del Congreso constitutivo de la Unión Argentina del Magisterio, la primera entidad de alcance nacional:

“...pues más de 100 delegados que traen representaciones de todos los rincones del país, desde el sur patagónico hasta el norte próximo, se han dado cita en esta provincia cordial [...] los congresales vienen con el mismo espíritu y sentimiento de los congresales de julio a decir la palabra consagratoria del sentir del magisterio argentino." (CUCUZZA, 2007. p. 126)

El 5 de noviembre de 1946 fueron sancionados gran parte de los asistentes al V Congreso Americano de Maestros y I Congreso de la Confederación Americana de Maestros, realizado en México en mayo del mismo año; y entre ellos fue dejada cesante en su cargo de maestra la Secretaria Tesorera de la UAM: Anunciada Mastelli.

No volvería al aula hasta 10 años después cuando tomó paralelamente a su grado primario las clases de didáctica y observación de 
clases de la Escuela Normal del Instituto "Esteban Echeverría" de Munro, Pcia. de Buenos Aires.

\section{Marea y contramarea: la querella de los métodos}

Para situar el contexto de recepción del "método Decroly" nos apoyamos en Roberta Paula Spregelburd cuando analiza el dictamen que formulara la Comisión de Textos de Lectura y Escritura del concurso de 1907, y advierte que el significado de la expresión "libro de lectura" sólo comienza a adquirir precisión una o dos décadas más tarde.

"Su definición era construida por la comisión por oposición a otros géneros textuales rechazados, algunos de los cuales son mencionados explícitamente (catecismos, novelas, cuentos, antologías,) y otros podemos leerlos entre líneas (ensayos, prensa)." (SPREGELBURD, 2012, pp. 206-212)

En cuanto al método, María Cristina Linares señala el surgimiento de "una nueva generación de libros de lectura" a partir del texto de Andrés Ferreira "El Nene", cuyo libro primero de 1895 mereció 120 ediciones:

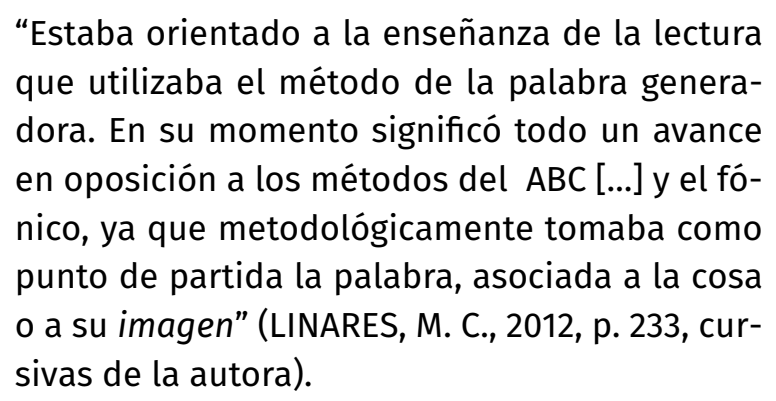

En ese contexto de predominio de palabra generadora, en un reciente trabajo, Susana Noemí Vital y Roberta Paula Spregelburd, se proponen analizar la introducción del método global para la enseñanza de la lectura en Argentina entre 1930 y $1970 .^{7}$

7 Vale señalar que Paulo Freire utiliza palabra generadora. También la aplica DINEA en la Campaña de Reactivación Educativa de Adultos para la Reconstrucción, en 1973 en Argentina. Véase BOTTARINI, 2012, pp. 494-501.
Comienzan con el libro de Emilia Dezeo y Juan M. Muñoz titulado “La enseñanza del lenguaje gráfico. Método natural para enseñar a leer y escribir publicado por Ferrari Hnos. en 1936". Registran "el relato de las experiencias realizadas entre 1932 y 1935 en una escuela de la Capital Federal en la que Emilia Dezeo cumplía funciones directivas [...] la escuela № 17 del Distrito Escolar 16 de la Capital Federal; el método fue aplicado por la Sra. Lucila P. de Garrido entre 1932 y 1934, y por las Srtas. María Isabel Risso de Moreo y Amelia de Agüero en 1935 , todas ellas maestras de primer grado inferior." (VITAL y SPREGELBURD, 2016, p. 8).

Dezeo y Muñoz se diferencian de Decroly señalando que "su método de lectura no es más que el método de Jacotot, con el agregado de las loterías y otros juegos que ya hemos visto se utilizaron desde los romanos." (Ibid., p. 9).

Vital y Spregelburd muestran a Clotilde Guillén de Rezzano desde la editorial Kapelusz, diferenciándose de los anteriores con una intensa difusión de Decroly traduciendo autores extranjeros como Charles Hendríx en "Cómo enseñar a leer por el método global" (1952) y "La enseñanza de la lectura por el método global" de Segers (1954 2a edición), autores enrolados en el escolanovismo; así como sus propias obras dedicadas a la formación de maestros como las didácticas generales y especiales y a la promoción de los centros de interés.

"A todo ello se suma la elaboración del libro de lectura para primer grado "Mamita" publicado por la misma editorial, compuesto desde la primera lectura por frases a las que les atribuye sentido, excluyendo a lo largo de todo el libro las letras y sílabas aisladas". (Ibid., p. 12).

Guillén de Rezzano utiliza el método de palabras generadoras como antecedente del global al señalar:

"El doctor Decroly aplicó la globalización a todas las fases de la técnica informativa [sic]. Es 
este el aspecto que más se ha generalizado y el que mejor se conoce. Su sistema de lectura ideo-visual, es fácilmente accesible a los maestros argentinos que desde El Nene, pasando por otros excelentes sistemas de lectura, han practicado, siempre, el sistema sintético analítico" (GUILLÉN de REZZANO, La Obra del Doctor Ovidio Decroly. En MEC., 1932, p. 152).

Vital y Spregelburd, consideran luego a Luis F. Iglesias en su desempeño como maestro en una escuela rural unitaria del partido de Esteban Echeverría en la provincia de Buenos Aires durante veinte años desde 1938. Iglesias (un "freinetiano" que confesaba que si hubiera leído a Freinet jamás hubiera realizado su experiencia en su escuela), cercano al Partido Comunista cuando más tarde dirige el periódico Educación Popular, destaca las virtudes del método. Dice así: “de las técnicas ensayadas, ninguna como el método global nos han ayudado a dar respuestas concretas a estos problemas del trabajo de primer grado Inferior. Este método que "ha sido reinventado varias veces» $y$ que seguiremos reinventando por mucho tiempo los maestros de primeras letras, encaja perfectamente en nuestras actividades". (Luis F Iglesias, "La escuela Rural Unitaria", citado por VITAL y SPREGELBURD, Op. Cit. p. 12).

Y para agregar complejidad, las autoras mencionadas, reseñan a Berta Perelstein de Braslavsky, enrolada en el Partido Comunista sumamente crítica de Decroly en el clásico libro "La querella de los métodos en la enseñanza de la lectura. Sus fundamentos psicológicos y la renovación actual".

El libro contiene un anexo con una revisión crítica de "la querella" realizado por su autora donde reafirma sus opiniones sobre el método Decroly cuarenta años después: "En la querella...se ponen de relieve los funda-
mentos irracionalistas de los principios elabo-
rados por Decroly. Se condena la "mística" y el
"milagro del interés" que busca sus fuentes en
los "instintos individuales" que incluyen a la inteligencia, y en los sociales, el "instinto grupal". En el concepto de "globalización" que elude el proceso de análisis y síntesis, se encuentra esa "especie de intuición intelectual" de la inmediatez que "capta ideas" gracias a ciertas "condiciones intrínsecas" que surgen "repentinamente" (BRASLAVSKY, 2014, p. 212)

Así como se pueden encontrar referencias a la filiación comunista de Iglesias y Braslavsky es posible hallar referencias a la filiación peronista de Dezeo y Clotilde Guillén de Rezzano; aunque se "cruzan" las pertenencias políticas con las posiciones sobre el método global quitando autorización para establecer relaciones lineales.

\section{Anotaciones para un amarre provisorio}

Finalmente Caruso cierra su trabajo con una interpelación a los historiadores de la educación reclamando una mayor investigación de las experiencias de la escuela nueva y su trayectoria social "para poder afirmar si tiene puerto, si los alcanzó o si el barco se ha echado alguna vez a navegar (CARUSO, 2010, p. 131)

Respecto del viaje de Decroly en 1915 no encontramos datos en El Monitor de la Educación Común de ese año que confirmen su presencia en Buenos Aires luego de revisar las secciones Sumario, Información Nacional, Información Extranjera, Revista de Revistas, Bibliografia y Sesiones del Consejo Nacional de Educación.

Agradecemos a los Dres. Marc Depaepe y Frank Simon por las informaciones sobre el tema:

El primero nos informó que, en la base de datos construida por Frank Simon, Ovide Decroly solicitó cuatro meses de licencia para ir a Colombia, desde el 1 de julio hasta fines de octubre de 1925; y que, "de acuerdo a nuestra base de datos" partió de Bélgica el 1 de julio y regresó en noviembre de 1925.

El segundo confirmó nuestras sospechas acerca de las dificultades de un viaje en ese 
año con Bélgica invadida por Alemania en la primera guerra mundial.

Procedimos a rastrear El Monitor de la Educación Común para el año 1925 para verificar los datos de Segers y la lejana posibilidad de combinar con un viaje al Río de la Plata su visita a Colombia, pero la revisión en El Monitor con los mismos criterios para 1915 no agregó ninguna información.

Para todo el año y consultando por autor la base de datos, sólo se encuentra un solo registro para "Decroly" correspondiente al clásico "Hacia una escuela renovada". Ediciones de la lectura. Madrid.1922.

Finalmente, y ello es muy significativo, no menciona visita alguna la conferencia "La Obra del Doctor Ovidio Decroly" leída por Clotilde Guillén de Rezzano en el acto de homenaje a su memoria realizada por la Sección Argentina de la Liga Internacional para la Educación Nueva el día 20 de setiembre de 1932 (Decroly había fallecido el 12 de setiembre). (GUILLÉN de REZZANO, 1932).

Con la "señora de Rezzano" (como la menciona el escriba del Monitor en la nota del cierre de los cursos que antes reseñamos) se vuelve a escuchar a "Clotilde Guillén"; y en esta conferencia se ocupa de fustigar a los "veinte y tantos siglos de academicismo pedagógico con un formulario de recetas anacrónicas y pedantes", al "verbalismo hueco, sin conexión con las necesidades de la vida", a "los timoratos, los eternos desconfiados de

\section{Referencias}

ALI JAFELLA, Sara. Escuela nueva en Argentina y Brasil: retrospectiva de un ideario pedagógico en la formación docente. Rev. Ped., Caracas, v. 23, n. 67, p. 333-344, may. 2002.

ALI JAFELLA, Sara Un ideario pedagógico en la formación superior. En: BIAGINI, Hugo E.; ROIG, Arturo A. (Directores). El pensamiento alternativo en la Argentina del siglo XX. Tomo I: Identidad, utopía, in- su propio criterio", a los "elementos aislados o grupos que se solidarizan [...] no para defender los intereses sagrados de los niños, sino para tomar por asalto posiciones [que] los representarían como un lastre que conviene eliminar..."

Registramos en este cierre estas duras expresiones de la escolanovista en el homenaje a Decroly porque, tan fuerte como el interrogante sobre si estuvo en Buenos Aires, lo fue el interrogante sobre qué ha sido en definitiva la escuela nueva.

Hemos visitado algunas respuestas, sin pretender agotarlas, tales como "la Escuela Nueva existiría, en primera instancia, como autoafirmación, como autoatribución de identidad" (CARUSO, 2001); "una forma de underground pedagógico" (ALI JAFELLA, 2004); "en la base de la nueva técnica del trabajo escolar está Ford y no Comenius" (PONCE, 1974); "la Escuela Nueva [desempeñó]la función de recomponer los mecanismos de hegemonía de la clase dominante" (SAVIANI en Caruso, 2001); "Los discursos de la escuela nueva en la Argentina construyeron una visión de la infancia caracterizada por el reconocimiento del alumno a partir de su identidad de niño, supuestamente invisibilizada por el normalismo de corte positivista" (CARLI, 2004);

Por nuestra parte, por ahora, sólo nos permitimos compartir el recuerdo de una nave que iniciaba su viaje cada vez que cruzábamos la puerta de un aula.

tegración (1900-1930). Buenos Aires: Biblos. 2004. p. 355-362.

BOTTARINI, Roberto Aníbal. Alfabetización de adultos y sujetos políticos (c.1960-1970). En: CUCUZZA, Héctor Rubén; SPREGELBURD, Roberta Paula. (Dir..). Historia de la lectura en Argentina. Del catecismo colonial a las netbooks estatales. Buenos Aires: Editoras del Calderón, 2012. p. 471-506. 
BRASLAVSKY, Berta P. de. La querella de los métodos en la enseñanza de la lectura. Sus fundamentos psicológicos y la renovación actual. Gonnet: UNIPE, 2014.

CARLI, Sandra. Niñez, pedagogía y política. Transformaciones de los discursos acerca de la infancia en la historia de la educación argentina entre 1880 y 1955. Buenos Aires: UBA; Miño y Dávila, 2003.

CARLI, Sandra. Escuela Nueva, cultura y política. En: BIAGINI, H. E.; ROIG, A. A. (Directores). El pensamiento alternativo en la Argentina del siglo XX. Tomo I: Identidad, utopía, integración (1900-1930). Buenos Aires: Biblos. 2004. p. 363-372.

CARUSO, Marcelo. ¿Una nave sin puerto definitivo? Antecedentes, tendencias e interpretaciones alrededor del movimiento de la Escuela Nueva. En: PINEAU, Pablo; DUSSEL, Inés; CARUSO, Marcelo R. (Comp.). La escuela como máquina de educar: tres escritos sobre un proyecto de la modernidad. Buenos Aires, Paidós, 2001. p. 93-134.

CRESPI, Graciela. La huelga docente de 1919 en Mendoza. En: MORGADE, Graciela. (Comp.). Mujeres en la educación. Género y docencia en la Argentina (1870-1930). Buenos Aires: Miño y Dávila Editores, 1997. p. $151-174$.

CUCUZZA, Héctor Rubén. Allons enfants de la patrie: 0 arquivo pessoal de Anunciada Mastelli. En: VENANCIO MIGNOT, Ana Chrystina; SANTOS CUNHA, María Teresa. (Orgs.). Práticas de memória docente. São Paulo: Cortez Editora, 2003. Hay versión castellana en Archivos de Ciencias de la Educación, Año 1, n. 1, La Plata: Departamento de Educación. Facultad de Humanidades, Universidad de La Plata, 2007. Disponible en: <http://www.archivosdeciencias. fahce.unlp.edu.ar/article/view/ARCHv01n01a05> Acceso en: 4 nov 2016.

CUCUZZA, Héctor Rubén. El Proyecto Histelea: nuevas aperturas teóricas y desafios metodológicos. Revista Internacional de Investigación en Educación, Colombia, Magis; Universidad Javeriana, v.4,n7., p. 45-46, mês jul-dic . 2011. Disponible en: <http:// revistas.javeriana.edu.co/index.php/MAGIS/article/ view/3555>. Acceso en: 4 nov. 2016.
DÁVILA BALSERA, Paulí; NAYA GARMENDIA, Luis María. La evolución de los derechos de la infancia: una visión internacional. Kingston: Encounters on Education. v. 7. Ontário: Faculty of Education, Queen's University, 2006.

DEPAEPE, Marc; SIMON, Frank. La diseminación de ideas deweyanas en Bélgica a través de los manuales de pedagogía. Historia Caribe, Barranquilla (Col), v. 4, n. 10, p. 7-17, 2005.

DEPAEPE, Marc; SIMON, Frank; VAN GORP, Angelo. El evolucionismo y el desarrollo del pensamiento educacional en Bélgica antes de la segunda Guerra Mundial. Revista Brasileira de História da Educação, Campinas, SP, v. 10, n. 24. p. 11-34. set./dez. 2010.

DUSSEL, Inés. Victor Mercante y la producción de un discurso científico sobre la educación. La Plata: Revista Archivos de Ciencias de la Educación, Departamento de Educación, Facultad de Humanidades, Universidad Nacional de La Plata, Año 8, n. 8, 4o Época, 2014.

FILHO, LourençO. Introducción al estudio de la escuela nueva. Buenos Aires: Kapelusz, 1964.

FONTANA, Esteban. La escuela media mendocina entre 1864 y 1930. En: PUIGGRÓS, Adriana. (Dirección). La educación en las Provincias y Territorios Nacionales (1885-1945). Buenos Aires: Galerna, 1993. p. 239-397.

FRECHTEL, Ignacio. La impronta taylorista en la filosofía de la educación escolanovista argentina: el caso de la "Reforma Rezzano" (1918-1925). En: CONGRESO LATINOAMERICANO DE FILOSOFÍA DE LA EDUCACIÓN, "La filosofía de la educación: tradición y actualidad", 2., 2013, Montevideo. Anais... Montevideo: Universidad de la República, 2013. (mimeo inédito).

GONÇÁLVES Vidal, Diana. (Org.). Dermeval Saviani: pesquisador, professor e educador. Belo Horizonte: Autores Associados, 2011.

GVIRTZ, Silvina. (Comp.). Escuela Nueva en Argentina y Brasil. Visiones comparadas. Buenos Aires: IICE; Miño y Dávila Editores, 1996.

GVIRTZ, SILVINA. Del curriculum prescripto al curriculum enseñado. Buenos Aires: Aique, 1997. 
HERRERA CORTÉS, Martha Cecilia. Modernización y escuela nueva en Colombia: 1914-1951. Santa Fé de Bogotá: Plaza \& Janes Editores Colombia, 1999.

LINARES, María Cristina. Nacimiento y trayectoria de una nueva generación de libros de lectura escolar: “El Nene" (1895-1956). En: CUCUZZA, Héctor Rubén; SPREGELBURD, Roberta Paula. (Dir.). Historia de la lectura en Argentina. Del catecismo colonial a las netbooks estatales. Buenos Aires: Editoras del Calderón, 2012. p. 215-253.

LIONETTI, Lucía. Víctor Mercante: agente político e intelectual del campo educativo en la Argentina de principios del siglo XX. Prohistoria, Rosario, Argentina, año X, n. 10, p. 93-112, prim. 2006.

MARÍN IBÁÑEZ, Ricardo. Los ideales de la escuela nueva. Revista de Educación, Madrid, Secretaría General Técnica/Centro de Publicaciones; Ministerio de Educación y Ciencia, n. 242, p. 23-42, 1976.

OBREGÓN, Javier Sáenz; SALDARRIAGA, Oscar; OSPINA, Armando. Mirar la infancia: pedagogía, moral y modernidad en Colombia. 1903-1946. Bogotá, Medellín: Colciencias; Ediciones Foro Nacional por Colombia; Ediciones Uniandes; Editorial Universidad de Antioquia, 1997.

OSTROVSKY, Ana Elisa. La sociedad de psicología en Argentina (1908-1913). Treinta y nueve hombres y una mujer. Revista de Historia de la Psicología, Valencia, Publicaciones de la Universitat de Valencia, v. 29 , n. 2, p. 55-67, 2008.

POZO ANDRÉS, María del Mar del. Desde L'Ermitage a la Escuela Rural Española: introducción, difusión y apropiación de los "centros de interés" decrolyanos (1907-1936). Revista de Educación, Ministerio de Educación y Ciencia, Madrid, número extraordinario, p. 143-166, 2007.

PONCE, Aníbal. Educación y lucha de clases. Buenos Aires: Cartago, 1974. (Obras Completas, vol. 3.

PUIGGRÓS, Adriana. Sujetos, disciplina y curriculum, 1885-1916. Buenos Aires: Galerna, 1990.

PUIGGRÓS, Adriana. Sociedad civil y estado. Buenos Aires: Galerna, 1991.
PUIGGRÓS, Adriana. Escuela, democracia y orden (1916-1943). Buenos Aires: Galerna, 1992.

PUIGGRÓS, Adriana. El lugar del saber: conflictos y alternativas entre educación, conocimiento y política. Buenos Aires: Galerna, 2003.

RÍOS BELTRAN, Rafael. Escuela Nueva y saber pedagógico en Colombia: apropiación, modernidad y métodos de enseñanza. Primera mitad del siglo XIX. Historia y Sociedad, [S.l.], n. 24, p. 79-107, 2013. Disponible en: <http://www.revistas.unal.edu.co/index.php/hisysoc/article/view/39709/47838>. Acceso en: 15 dec. 2016.

SÁNCHEZ LUQUEZ, Elizabeth. La experiencia de escuela nueva en Mendoza. Revista Historia de la Educación Latinoamericana, Tunja-Boyacá, Universidad Pedagógica y Tecnológica de Colombia, Rhela. Disponible en: <http://revistas.uptc.edu.co/ index.php/historia educacion latinamerican/article/viewFile/2771/2711>. Acceso en: 22 nov. 2016.

SEGERS, J. A. En torno a Decroly (La psichologie de l'enfant normal et anormal d'apres le Dr Decroly). Traducción, presentación y bibliografía de Jordi Mones y Pujol-Busquets. Madrid: Ministerio de Educación y Ciencia, 1985.

SPREGELBURD, Roberta Paula ¿Qué se puede leer en la escuela? El control estatal del texto escolar (1880-1916). En: CUCUZZA, Héctor Rubén; SPREGELBURD, Roberta Paula. (Dir.). Historia de la Lectura en Argentina. Del catecismo colonial a las netbooks estatales. Buenos Aires: Editoras del Calderón, 2012. p. 171-214.

SUASNÁBAR, Claudio; CHELI, Verónica. El papel de los Congresos Internacionales en el proceso de conformación del campo disciplinario de la educación en Argentina (1910-1937). Revista Brasileira de História da Educação, Sociedade Brasileira de História da Educação, Maringá, PR. v. 15, n. 3, p. 83-107, set./dez. 2015.

VITAL, Susana Noemí; SPREGELBURD, Roberta Paula. La enseñanza inicial de la lectura por el método global en Argentina. Entre la prescripción y la práctica (1930-1979 circa). Revista Polifonías, Luján, Departamento de Educaciòn de la Universidad Na- 
cional de Luján, Año V, n. 9, p.100-125, 2016. Disponible en: http://www.polifoniasrevista.unlu.edu.ar/ sites/www.polifoniasrevista.unlu.edu.ar/files/site/ Polifonias-9.pdf

\section{Fuentes}

ACUÑA, Ángel. Discurso pronunciado en el acto del sepelio del Dr. Emilio Giménez Zapiola. El Monitor de la Educación Común. Año 50. № 697. Buenos Aires: Biblioteca Nacional del Maestro. Ministerio de Educación. Reservorio: Sala del Tesoro. 1931. Disponible en: <http://www.bnm.me.gov.ar/ebooks/reader $/$ reader .php? $m o n=1 \& v t=n \& d i r=00150907 \&$ num img=3\&num fin=21>. Acceso en: 14 dic. 2016.

ACUÑA, Ángel. Sistema Decroly (2) (continuación del número anterior). El Monitor de la Educación Común. Año 54. № 744. Buenos Aires: Biblioteca Nacional del Maestro. Ministerio de Educación. Reservorio: Sala del Tesoro. 1934. Disponible en: <http:// www.bnm.me.gov.ar/ebooks/reader/reader.php?mon $=1 \& v t=n \& d i r=00150919 \& c=0003 \&$ num_img=3\&num_fin=11>. Acceso en: 10 nov. 2016.

DABAT de LÓPEZ ELYCHERI, Bernardina. Apuntes sobre una adaptación del Método "Decroly" a nuestras prácticas escolares. El Monitor de la Educación Común. Año 52. № 717. Buenos Aires: Biblioteca Nacional del Maestro. Ministerio de Educación. Reservorio: Sala del Tesoro. 1932. Disponible en: <http:// www.bnm.me.gov.ar/ebooks/reader/reader.php?mon $=1 \& v t=n \& d i r=00150912 \& c=0006 \&$ num img=9\&num_fin=17>. Acceso en: 10 nov. 2016.
La investigación en el Consejo Nacional de Educación. El Monitor de la Educación Común. Año 28. № 427. Buenos Aires: Biblioteca Nacional del Maestro. Ministerio de Educación. Reservorio: Sala del Tesoro. 1908. Disponible en: <http://www.bnm.me. gov.ar/ebooks/reader/reader.php?mon=1\&vt=n\&dir $=00150824 \&$ num_img $=480 \&$ \&um_fin $=500>$. Acceso en: 15 nov. 2016.

Los cursos de Escuela Activa para maestros. El Monitor de la Educación Común. Año 52. No. 721. Buenos Aires: Biblioteca Nacional del Maestro. Ministerio de Educación. Reservorio: Sala del Tesoro. 1933. Disponible en: <http://www.bnm.me.gov.ar/ ebooks/reader/reader.php?dir=00150914\&num img $=00150914 \_0098-00 \& m o n=1 \& v n=s \& v i=s \& v t=n \&$ $\mathrm{vp}=\mathrm{s} \& \mathrm{vv}=\mathrm{s} \& \mathrm{vh}=\mathrm{s} \& \mathrm{modo}=\& z \mathrm{zom}=100 \& \mathrm{c}=\& \mathrm{buscar}=$ \&ir_a $a=100 \& b u s c a r=\mid r+a+p \% E 1 g>$. Acceso en: 17 nov. 2016.

REZZANO, Clotilde Guillén de. La obra del Dr. Ovidio Decroly. El Monitor de la Educación Común. Año 34. No. 514.Tomo 55. Buenos Aires: Biblioteca Nacional del Maestro. Ministerio de Educación. Reservorio: Sala del Tesoro. 1932. Disponible en: <http:// www.bnm.me.gov.ar/ebooks/reader/reader. php?dir $=00150912 \&$ num_img $=00150912 \_0146$ $00 \& m o n=1 \& v n=s \& v i=s \& v t=n \& v p=s \& v v=s \& v h=s \& c=0$ 006\&zoom $=150 \&$ modo $=>$.Acceso: 20 nov. 2016.

Recebido em: 06.03.2017 Aprovado em: 25.04.2017

\footnotetext{
Héctor Rubén Cucuzza Profesor Emérito del Departamento de Educación, Universidad Nacional de Luján - Buenos Aires, Argentina.e-mail: rubencucuzza@gmail.com

Universidad Nacional de Luján - Ruta 5 y Avenida Constitución - (6700) Luján, Buenos Aires, Argentina. Telefone: (54) (11) 46138695
} 